\title{
SYSTEMS OF FIXED POINT SETS
}

\author{
BY
}

\author{
A. D. ELMENDORF
}

\begin{abstract}
Let $G$ be a compact Lie group. A canonical method is given for constructing a $G$-space from homotopy theoretic information about its fixed point sets. The construction is a special case of the categorical bar construction. Applications include easy constructions of certain classifying spaces, as well as $G$-EilenbergMac Lane spaces and Postnikov towers.
\end{abstract}

0. Introduction. Let $G$ be a compact Lie group and $X$ a $G$-space. The equivariant homotopy theory of $X$ is reflected to a remarkable extent in its system of fixed point sets, defined as a functor from a certain category $O_{G}$ to Top, the category of topological spaces. (Our spaces will be compactly generated weak Hausdorff; they may or may not be equipped with a basepoint, depending on the context.)

These functors, or systems, have considerable technical advantages over $G$-spaces; it is easy to apply most homotopy theoretic constructions to them, whereas in many cases it is unclear how to proceed for $G$-spaces. It is the purpose of this paper to present a canonical way of recovering from any system a $G$-space which preserves all the homotopy theoretic structure of the system. This allows us to give easy equivariant versions of some standard topological constructions such as Eilenberg-Mac Lane spaces and Postnikov towers, and to simplify other equivariant constructions. ${ }^{1}$

1. Statements of the main theorems. Throughout, $G$ is a fixed compact Lie group.

Definitions. The category of canonical orbits, written $O_{G}$, is a topological category with discrete object space

$$
\left|O_{G}\right|=\{G / H: H \text { a closed subgroup of } G\}
$$

and morphisms the $G$-maps, topologized by requiring the natural bijection

$$
\operatorname{Hom}_{O_{G}}(G / H, G / K) \cong(G / K)^{H}
$$

to be a homeomorphism. By an $O_{G}$-space we shall mean a continuous contravariant functor from $O_{G}$ to Top; these functors form the objects of a topological category in the usual manner. We will also consider $O_{G}$-rings, $O_{G}$-groups, etc., defined similarly.

Definition. Let $X$ be a $G$-space. The fixed point set system of $X$, written $\Phi X$, is an $O_{G}$-space defined as follows:

$$
\Phi X(G / H)=X^{H}
$$

Received by the editors October 30, 1981 and, in revised form, April 26, 1982.

1980 Mathematics Subject Classification. Primary 57S10, 55N25.

Key words and phrases. Equivariant homotopy.

'Portions of this paper were presented to the Conference on Current Developments in Algebraic Topology, University of Western Ontario, July, 1981. 
and if $\theta: G / H \rightarrow G / K$ corresponds to $g K \in(G / K)^{H}$ under the correspondence (*), we define

$$
\Phi X(\theta)(x)=g x \in X^{H}
$$

for any $x \in X^{K}$. It is clear that $\Phi$ is a functor from $G$-spaces to $O_{G}$-spaces.

Definition. A $G$-map $f: X \rightarrow Y$ is a weak $G$-equivalence if $f_{*}: \pi_{n}\left(X^{H}\right) \rightarrow \pi_{n}\left(Y^{H}\right)$ is an isomorphism for all $n \geqslant 0$ and all closed subgroups $H$. If $X$ and $Y$ are $G$-CW complexes, it follows that $f$ is a $G$-homotopy equivalence.

Definition. A $C W-O_{G}$-space is an $O_{G}$-space $T$ such that each space $T(G / H)$ is a CW-complex and each structure map $T(G / H) \rightarrow T(G / K)$ is cellular. We will call $T$ regular if it is homotopy equivalent (in the sense detailed below) to a $\mathrm{CW}-\mathrm{O}_{G}$-space.

Definition. Let $T, U$ be $O_{G}$-spaces. Define $T \times I$ to be the $O_{G}$-space given by the composite functor $O_{G} \stackrel{T}{\rightarrow}$ Top $\stackrel{\times I}{\rightarrow}$ Top. There are the usual maps $i_{0}, i_{1}$ from $T$ to $T \times I$, and we say two maps $f, g$ from $T$ to $U$ are homotopic if the diagram

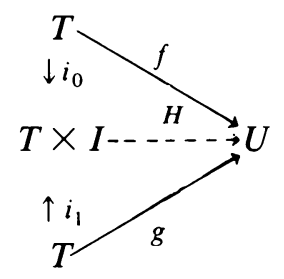

can be filled by the homotopy $H$. As usual, this gives rise to a homotopy category of $O_{G}$-spaces; we denote the set of homotopy classes by $[T, U]_{O_{G}}$.

THEOREM 1. There is a functor $C: O_{G}$-spaces $\rightarrow G$-spaces and a natural transformation $\eta: \Phi C \rightarrow$ id such that for each $O_{G}$-space $T$ and each $H, \eta:(C T)^{H} \rightarrow T(G / H)$ is a homotopy equivalence (it is actually a strong deformation retract). If $T$ is regular, then $C T$ has the G-homotopy type of a G-CW complex.

COROLlary. If $X$ is a $G$-space, there is a natural weak G-equivalence from $C \Phi X$ to $X$ obtained by restricting $\eta$ to $G /\{e\}$.

Let $[X, Y]_{G}$ denote the set of $G$-homotopy classes of maps from $X$ to $Y$.

TheOrem 2 (MCCLURE). Let $X$ be a G-space which is G-equivalent to a $G$-CW complex, and let $T$ be a regular $O_{G}$-space. Then there is a natural bijection

$$
[X, C T]_{G} \cong[\Phi X, T]_{o_{G}} \text {. }
$$

2. Applications. We first consider classifying spaces for families. A family of subgroups of $G$ is a set closed under conjugation and subgroups. If $\mathscr{F}$ is a family and $X$ is a $G$-space, we say $X$ is $\mathscr{F}$-isotropic if every isotropy subgroup $G_{x}$ for $x \in X$ is in $\mathcal{F}$. Following tom Dieck [2, §7.2], we define a classifying space for $\mathcal{F}$ to be an $\mathscr{F}$-isotropic $G$-space $B \mathscr{F}$ such that for any $X$ which is $\mathscr{F}$-isotropic, $[X, B \mathscr{F}]_{G}$ consists of a unique element. We can easily construct $B \mathscr{F}$ as follows. Let $T$ be the $O_{G}$-space in which

$$
T(G / H)= \begin{cases}* & \text { if } H \in \mathscr{F} \\ \varnothing & \text { if } H \notin \mathscr{F}\end{cases}
$$


Then it is a direct corollary of Theorem 2 that $C T$ is a classifying space for $\mathscr{F}$. In the special case where $\mathscr{F}$ consists of the trivial subgroup $\{e\}$ only, this turns out to be the usual bar construction for $E G$.

Smith theory. Assume for the moment that $G$ is a finite $p$-group. It is easy to construct an $O_{G}$-space $T$ in which $T(G /\{e\})=S^{n}$ and $T(G / G)$ is arbitrary (say $\left.C P^{\infty}\right)$. Then $C T$ is a homotopy sphere on which $G$ acts with fixed point set homotopic to $\mathbf{C} P^{\infty}$ (or whatever). This does not contradict Smith theory because $C T$ is always infinite dimensional.

G-connected covers. In ordinary homotopy theory, when we wish to pass to a connected space from a general pointed space, we look at the basepoint component. Equivariantly, we wish to do this simultaneously on all fixed point sets, which is impossible. We can do it up to homotopy, though, as follows. Let $X$ be a pointed $G$-space with base point in $X^{G}$. The system $\Phi X$ then takes values in Top ${ }_{+}$, the category of based spaces. Define $T_{0}(X)$ to be the $O_{G}$-space given by the composite $O_{G} \stackrel{\Phi X}{\rightarrow} \mathrm{Top}_{+} \stackrel{R}{\rightarrow}$ Top $_{+}$, where $R$ is restriction to basepoint components, and let $X_{0}=C T_{0}(X)$. The natural transformation $R \dot{\rightarrow}$ id given by inclusion of the basepoint component induces an $O_{G}$-map $T_{0}(X) \rightarrow \Phi X$, so we get a natural map

$$
X_{0}=C T_{0}(X) \rightarrow C \Phi X \stackrel{D \eta}{\rightarrow} X
$$

which is, up to homotopy, inclusion of the basepoint component on each fixed point set. The map $D \eta$ is the weak $G$-equivalence of the corollary to Theorem 1 .

Eilenberg-Mac Lane spaces. Let $\lambda$ be an $O_{G}$-group, $n$ an integer with $n \geqslant 1$. If $n>1$, we require $\lambda$ to be Abelian. An Eilenberg-Mac Lane $G$-space of type $(\lambda, n)$ is a $G$-space $X$ of the $G$-homotopy type of a $G$-CW complex such that, for each $H, X^{H}$ is a $K(\lambda(G / H), n)$, and the composite $O_{G} \stackrel{\Phi X}{\rightarrow}$ Top $\stackrel{\pi_{n}}{\rightarrow}$ Grps coincides with $\lambda$. These are the classifying spaces for Bredon cohomology; see $[1,3]$. Such $G$-spaces can be constructed functorially on $\lambda$ as follows. Let $B^{n}$ be a functorial construction of ordinary Eilenberg-Mac Lane spaces such as the iterated bar construction. Then the composite $O_{G} \stackrel{\lambda}{\rightarrow}$ Grps $\stackrel{B^{n}}{\rightarrow}$ Top is an $O_{G}$-space, and $C\left(B^{n} \circ \lambda\right)$ is the desired Eilenberg-Mac Lane $G$-space.

Postnikov towers. Equivariant Postnikov towers are just like ordinary Postnikov towers except that they use the equivariant Eilenberg-Mac Lane spaces referred to above. Using the obstruction theory arising from the use of the bar construction in the definition of $C$, we can construct such Postnikov towers for nilpotent $G$-spaces; the details are in $\S 6$. Postnikov towers have also been constructed by Triantafillou in [6] by completely different methods in the case where $G$ is a finite group.

3. Proof of Theorem 1. Let $T$ be an $O_{G}$-space. We must construct the $G$-space $C T$ and the natural map $\eta:(C T)^{H} \stackrel{\cong}{\rightarrow} T(G / H)$.

Let $J: O_{G} \rightarrow$ Top be the covariant functor which assigns to $G / H$ its underlying space and to a $G$-map its underlying map. (If we are considering pointed spaces, attach a disjoint basepoint and use smash products in the geometric realization below.) We may then form the bar complex $B_{*}\left(T, O_{G}, J\right)$; this is a simplicial space 
in which $B_{n}\left(T, O_{G}, J\right)$ consists of $(n+2)$-tuples $\left(t ; f_{1}, f_{2}, \ldots, f_{n} ; c\right)$ where the $f_{i}$ 's are composable arrows in $O_{G}$, say $f_{i}: G / H_{i} \rightarrow G / H_{i-1}$, and $t \in T\left(G / H_{0}\right), c \in G / H_{n}$. The boundaries are given as follows:

$$
\begin{aligned}
& \partial_{0}\left(t ; f_{1}, \ldots, f_{n} ; c\right)=\left(f_{1}^{*}(t) ; f_{2}, \ldots, f_{n} ; c\right), \\
& \partial_{n}\left(t ; f_{1}, \ldots, f_{n} ; c\right)=\left(t ; f_{1}, \ldots, f_{n-1} ;\left(f_{n}\right)_{*}(c)\right),
\end{aligned}
$$

and $\partial_{i}$ for $0<i<n$ is given by composing the appropriate pair of $f$ 's. Degeneracies are the insertion of identity maps in the appropriate spots. (This is a special case of the general construction given in [5, §12].) The group $G$ acts simplicially on $B_{*}\left(T, O_{G}, J\right)$ through its action on the coset coordinate, and consequently the geometric realization $B\left(T, O_{G}, J\right)$ is a $G$-space. We define

$$
C T=B\left(T, O_{G}, J\right) \text {. }
$$

We next require the homotopy equivalence $\eta:(C T)^{H} \rightarrow T(G / H)$, natural in $H$. We have

$$
(C T)^{H}=B\left(T, O_{G}, J\right)^{H}=B\left(T, O_{G}, \operatorname{Hom}_{O_{G}}(G / H,-)\right) ;
$$

the second equality follows from the bijection (*) and the fact that $G$ acts on the last coordinate only. Now it is a general property of the bar construction that for any

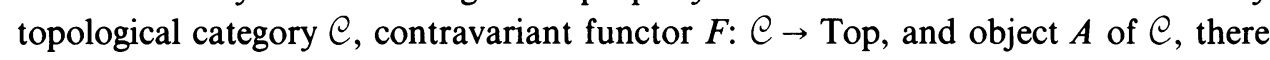
is a natural map

$$
\eta: B\left(F, \bigodot, \operatorname{Hom}_{\bigodot}(A,-)\right) \rightarrow F(A)
$$

which is a strong deformation retraction. This map is induced by a simplicial map

$$
\eta_{*}: B_{*}\left(F, \bigodot, \operatorname{Hom}_{\mathcal{C}}(A,-)\right) \rightarrow F(A)_{*},
$$

where $F(A)_{*}$ is the simplicial space all of whose components are $F(A)$ and all of whose face and degeneracy maps are the identity. In our case, $\eta_{*}$ is given by the formula

$$
\eta_{n}\left(x ; f_{1}, \ldots, f_{n} ; f\right)=\left(f_{1} \circ \ldots \circ f_{n} \circ f\right)^{*}(x),
$$

where $f$ is an element of $\left(G / H_{n}\right)^{H}=\operatorname{Hom}_{O_{G}}\left(G / H, G / H_{n}\right)$. The proof that $\eta$ is a strong deformation retraction is a standard simplicial argument contained in [5].

4. Adjunction relations and the proof of Theorem 2. Let $T$ be an $O_{G}$-space. Since the space of $O_{G}$-endomorphisms of $G /\{e\}$ is precisely $G$, it follows that $T(G /\{e\})$ is a $G$-space, which we denote by $D T$. It is easy to see that $D$ is left adjoint left inverse to $\Phi$, and the naturality of $\eta$ implies that $D \eta: C T \rightarrow T(G /\{e\})=D T$ is a $G$-map. It also follows, since $\Phi D \eta=\eta$ on $\Phi X$, that $D \eta: C \Phi X \rightarrow X$ is a weak $G$-equivalence and, consequently, a $G$-equivalence if $X$ is $G$-equivalent to a $G$-CW complex. Note also that $\Phi, C$, and $D$ preserve homotopies. We can now explicitly formulate the bijection given in Theorem 2. Suppose $f: X \rightarrow C T$ and $s: \Phi X \rightarrow T$ are maps in the appropriate categories. We define

$$
\alpha(f)=\eta \circ \Phi f, \quad \beta(s)=C s \circ(D \eta)^{-1},
$$


and the claim is that $\alpha$ and $\beta$ are inverse to each other on homotopy sets. (This proof is derived from Jim McClure's original proof given in [4].) To see that $\alpha \beta(s) \simeq s$, we examine the square

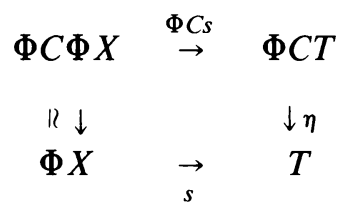

Here the unlabeled arrow is $\eta$ or $\Phi D \eta$; they coincide on $\Phi X$. The square commutes by naturality of $\eta$, showing

$$
s \simeq \eta \circ \Phi\left(C s \circ(D \eta)^{-1}\right)=\alpha \beta(s) .
$$

(We have also used the fact that $C, D$, and $\Phi$ preserve homotopies.)

For the converse, we look at

\begin{tabular}{|c|c|c|c|}
\hline$C \Phi X$ & $\stackrel{C \Phi f}{\rightarrow}$ & СФСТ & $\stackrel{C \eta}{\rightarrow}$ \\
\hline$\eta \downarrow D \eta$ & & $\ell \downarrow D \eta$ & \\
\hline$X$ & $\vec{f}$ & $C T$ & \\
\hline
\end{tabular}

The square commutes by naturality of $D \eta$, so we get

$$
\beta \alpha(f)=C(\eta \circ \Phi f) \circ(D \eta)^{-1}=C \eta \circ(D \eta)^{-1} \circ f .
$$

Since both $C \eta$ and $D \eta$ are homotopy equivalences, this shows $\beta \alpha$ is a bijection. But since $\alpha \beta=\mathrm{id},(\beta \alpha)^{2}=\beta \alpha$, so $\beta \alpha=$ id also.

5. Obstructions and $G$-maps. In this section we work with pointed spaces only. Let $X$ and $Y$ be $G$-spaces, $f: X \rightarrow Y$ a $G$-map. The equivariant homotopy class $[f]$ determines an ordinary homotopy class $\left[f^{H}\right] \in\left[X^{H}, Y^{H}\right]$ for each $H<G$, and it is natural to ask to what extent we can reverse the process: given classes $\left[f_{H}\right] \in$ [ $X^{H}, Y^{H}$ ], is there a $G$-map $f$ with $f^{H} \simeq f_{H}$ for all $H$ ? If so, is it unique? In highly favorable circumstances to be detailed below, the answer to both questions is yes.

Definition. A natural family is a set of homotopy classes $\left[f_{H}\right] \in\left[X^{H}, Y^{H}\right]$ such that the squares

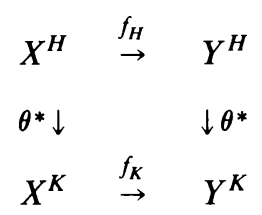

homotopy commute for all $\theta \in \operatorname{Hom}_{O_{G}}(G / K, G / H)$.

Clearly, the restrictions to fixed point sets from an equivariant homotopy class form a natural family.

TheOREM 3. Let $X$ and $Y$ be G-spaces with $X$ G-equivalent to a G-CW complex. Suppose further that, for each $K$ and $H$ :

(a) $\left[X^{K}, Y^{H}\right]$ is a group, and 
(b) $\left[X^{K}, \Omega^{n} Y^{H}\right]$ is trivial for $n \geqslant 1$.

Then restriction to fixed point sets induces a bijection between equivariant homotopy classes $[f] \in[X, Y]_{G}$ and natural families from $\Phi X$ to $\Phi Y$.

CoROllary. If $X$ is $a K(\lambda, n)$ and $Y$ is a $K\left(\lambda^{\prime}, n\right)$, then

$$
[X, Y]_{G} \cong \operatorname{Nat}\left(\lambda, \lambda^{\prime}\right)
$$

where $N$ at refers to the natural transformations from the functor $\lambda$ to the functor $\lambda^{\prime}$. In particular, $K(\lambda, n)$ 's are unique up to G-homotopy type.

Proof of Theorem 3. Since $X$ is $G$-equivalent to a $G$-CW complex, $D \eta$ : $C \Phi X \rightarrow X$ is a $G$-equivalence. The bar construction filtration on $C \Phi X$ has 0 th filtration $\bigvee_{H}\left(X^{H} \wedge(G / H)_{+}\right)$, and we have the following commutative diagram:

$$
\begin{array}{ccc}
{[X, Y]_{G}} & \stackrel{\rho}{\rightarrow} & \underset{H}{X}\left[X^{H}, Y^{H}\right] \\
(D \eta)^{*} \downarrow \| l & & \downarrow \| l \\
{[C \Phi X, Y]_{G}} & \underset{i^{*}}{\rightarrow} & {\left[{ }_{H}^{\vee}\left(X^{H} \wedge(G / H)_{+}\right), Y\right]_{G}}
\end{array}
$$

Here $\rho$ is restriction to fixed point sets, $i^{*}$ is induced by inclusion of the 0 th filtration, and the unlabelled isomorphism arises from the adjunction

$$
\left[X^{H} \wedge(G / H)_{+}, Y\right]_{G} \cong\left[X^{H}, Y^{H}\right]
$$

for each closed subgroup $H$. Elements $\left\{\left[f_{H}\right]\right\} \in X_{H}\left[X^{H}, Y^{H}\right]$ can be lifted along $\rho$ iff the corresponding lifting problem along $i^{*}$ can be solved; we attack the latter problem one filtration at a time.

Suppose we have a $G$-map $f$ from the $(n-1)$ st filtration of $C \Phi X$ to $Y$, and we wish to extend to the $n$th filtration. This is equivalent to filling all the diagrams

$$
X^{K} \wedge P_{+} \wedge(G / H)_{+} \wedge\left(\partial \Delta^{n}\right)_{+} \rightarrow X^{K} \wedge P_{+} \wedge(G / H)_{+} \wedge\left(\Delta^{n}\right)_{+}
$$

where $P$ is any product

$$
\operatorname{Hom}_{O_{G}}\left(G / H_{1}, G / H_{0}\right) \times \cdots \times \operatorname{Hom}_{O_{G}}\left(G / H_{n}, G / H_{n-1}\right)
$$

in which $H_{0}=K, H_{n}=H$. Letting $F($,$) denote the function space of basepoint$ preserving maps, standard adjunctions yield an equivalent diagram

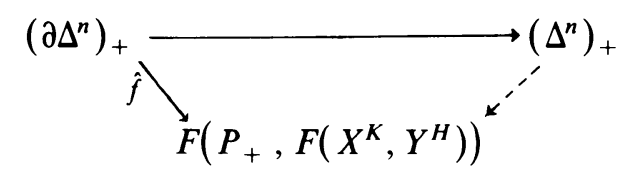

Now consider $\hat{f}$ as an element of $F\left(\left(\partial \Delta^{n}\right)_{+}, F\left(P_{+}, F\left(X^{K}, Y^{H}\right)\right)\right)$. This is the middle term of the fibration with section

$$
\begin{aligned}
F\left(\partial \Delta^{n}, F\left(P_{+}, F\left(X^{K}, Y^{H}\right)\right)\right) & \rightarrow F\left(\left(\partial \Delta^{n}\right)_{+}, F\left(P_{+}, F\left(X^{K}, Y^{H}\right)\right)\right) \\
& \underset{i}{\stackrel{\pi}{\rightleftarrows}} F\left(P_{+}, F\left(X^{K}, Y^{H}\right)\right)
\end{aligned}
$$


where $\pi$ is evaluation at the basepoint of $\partial \Delta^{n}$. Since $\Delta^{n}$ is contractible, the diagram fills iff $\hat{f}$ and $i \pi \hat{f}$ lie in the same component of the total space, which will be true iff they lie in the same component of the fiber over $\pi f$ due to the existence of the section. Since $\left[X^{K}, Y^{H}\right]$ is assumed to have group structure, we may take difference of components, giving us as obstruction a component of the fiber

$$
F\left(\partial \Delta^{n}, F\left(P_{+}, F\left(X^{K}, Y^{H}\right)\right)\right) \cong F\left(P_{+}, F\left(X^{K}, \Omega^{n-1} Y^{H}\right)\right) ;
$$

that is, the obstruction is an element of $\left[P_{+}, F\left(X^{K}, \Omega^{n-1} Y^{H}\right)\right]$. Now if $n>1$, $F\left(X^{K}, \Omega^{n-1} Y^{H}\right)$ is aspherical, since $\left[X^{K}, \Omega^{k} Y^{H}\right]=*$ for $k \geqslant 1$. This shows that $\left[P_{+}, F\left(X^{K}, \Omega^{n-1} Y^{H}\right)\right]=*$ for $n>1$, so once we extend $f$ to the first filtration, it will automatically extend to all of $C \Phi X$.

When $n=1, P=(G / H)^{K}$ and the problem is to fill the diagrams

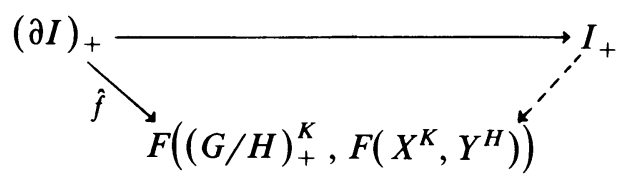

Since $\left[X^{K}, \Omega^{k} Y^{H}\right]=*$ for $k \geqslant 1$ and $\left[X^{K}, Y^{H}\right]$ has group structure, the projection

$$
F\left(X^{K}, Y^{H}\right) \rightarrow \pi_{0} F\left(X^{K}, Y^{H}\right)=\left[X^{K}, Y^{H}\right]
$$

is a weak equivalence, and consequently so is

$$
F\left((G / H)_{+}^{K}, F\left(X^{K}, Y^{H}\right)\right) \rightarrow F\left((G / H)_{+}^{K},\left[X^{K}, Y^{H}\right]\right) .
$$

Since $(\partial I)_{+} \rightarrow I_{+}$is a cofibration, it follows that the above diagram fills iff the derived diagram

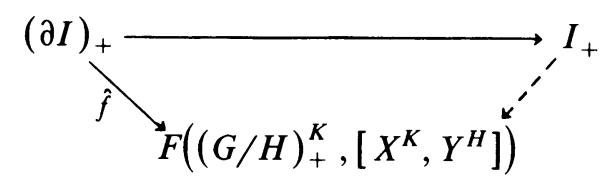

fills. An easy check then shows this to be equivalent to the homotopy commutativity of the diagrams

$$
\begin{array}{ccc}
X^{H} & \stackrel{f_{H}}{\rightarrow} & Y^{H} \\
\theta^{*} \downarrow & & \downarrow \theta^{*} \\
X^{K} & \overrightarrow{f_{K}} & Y^{K}
\end{array}
$$

which is precisely the condition that $f$ arise from a natural family. It follows that every natural family extends to an equivariant homotopy class.

To see that this class is unique, suppose $f$ and $g$ are $G$-maps from $X$ to $Y$ such that $f^{H} \simeq g^{H}$ for each $H$. This translates into a $G$-homotopy on the 0 th filtration of $C \Phi X$ which we wish to lift through the higher filtrations. Similar arguments now show that the obstructions to lifting to the $n$th filtration lie in $\left[P_{+}, F\left(X^{K}, \Omega^{n} Y^{H}\right)\right]$, which is trivial. Therefore, $f \simeq_{G} g$, and we are done. 
6. Postnikov towers for nilpotent $G$-spaces. In this section we use Theorem 3 to construct Postnikov towers. First we fix terminology. Let $X$ be a $G$-space. Then $\pi_{n}(X)$ is the composite

$$
O_{G} \stackrel{\Phi X}{\rightarrow} \text { Top } \stackrel{\pi_{n}}{\rightarrow} \text { Grps; }
$$

$\underline{\pi}_{1}(X)$ is an $O_{G}$-group and $\underline{\pi}_{n}(X)(n \geqslant 2)$ is an abelian $O_{G}$-group with $\underline{\pi}_{1}(X)$ - $O_{G}$-module structure.

Definition. A $G$-space $X$ is nilpotent if each $\pi_{n}(X), n \geqslant 1$, is nilpotent as an $O_{G}$-module over $\underline{\pi}_{1}(X)$, i.e., there are $O_{G}$-submodules

$$
\{\underline{0}\}=\underline{\pi}_{n, 0}(X) \subset \underline{\pi}_{n, 1}(X) \subset \cdots \subset \underline{\pi}_{n, r_{n}}(X)=\underline{\pi}_{n}(X)
$$

such that the subquotients $A_{n, j}=\underline{\pi}_{n, j+1}(X) / \underline{\pi}_{n, j}(X)$ are abelian with trivial $\underline{\pi}_{1}(X)$ action. This is equivalent to saying each $X^{H}$ is nilpotent in the usual sense with a uniform bound on the order of nilpotence in each dimension.

Definition. A Postnikov tower for $X$ is an inverse system of $G$-spaces $\cdots \rightarrow X_{2} \rightarrow$ $X_{1} \rightarrow X_{0}=*$ in which each $X_{j+1} \rightarrow X_{j}$ is the homotopy fiber of a $G$-map $X_{j} \rightarrow$ $K(\lambda, n)$ (where $n$ is a monotone function of $j$ ) together with a weak equivalence

$$
X \rightarrow \lim _{\leftarrow} X_{j} \text {. }
$$

Definition. A $G$-space $X$ is $G$-connected if each fixed point set $X^{H}$ is nonempty and connected.

TheOREM 4. Let $X$ be a nilpotent $G$-connected $G$-CW complex. Then $X$ has a Postnikov tower.

Proof. We can attach $G$-cells to $X$ to form $G$-spaces $X_{n, j}$ such that

$$
\underline{\pi}_{k}\left(X_{n, j}\right)= \begin{cases}\underline{\pi}_{k}(X), & k<n, \\ \underline{\pi}_{n}(X) / \underline{\pi}_{n, j}(X), & k=n, \\ \underline{0}, & k>n .\end{cases}
$$

This can be done by induction over the orbit types, since attaching $e^{n} \times G / H$ by gluing along $S^{n-1} \times G / H$ affects only $X^{K}$ with $(K) \leqslant(H)$, and since $\underline{\pi}_{n, j}(X)$ is a system, the classes killed in the $\pi_{n}\left(X^{K}\right)$ 's by attaching $e^{n} \times G / H$ will be among those we wish to kill in any case. By induction on the attached cells, we have $G$-maps $p_{u, j}: X_{n, j+1} \rightarrow X_{n, j}$ making

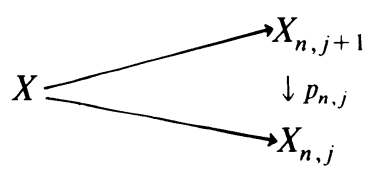

commutative, with the homotopy fiber $F p_{n, j}$ a $K\left(A_{n, j}, n\right)$.

Next we examine the fiber sequence on fixed point sets:

$$
K\left(A_{n, j}(G / H), n\right) \rightarrow X_{n, j+1}^{H} \stackrel{p_{n, j}^{H}}{\rightarrow} X_{n, j}^{H} .
$$


Since $\pi_{1}\left(X^{H}\right)$ acts trivially on $A_{n, j}(G / H), \pi_{1}\left(X_{n, j}^{H}\right)$ acts trivially as well, and we can classify this fibration by a map

$$
\hat{k}_{n, j}^{H}: X_{n, j}^{H} \rightarrow K\left(A_{n, j}(G / H), n+1\right)
$$

which factors through the cone of $p_{n, j}^{H}$, where $i_{n, j}^{H}$ is the canonical inclusion into the cone:

$$
\begin{array}{ccc}
\multicolumn{3}{c}{C p_{n, j}^{H}} \\
i_{n, j}^{H} \nearrow & \searrow \tilde{k}_{n, j}^{H} \\
X_{n, j}^{H} & \underset{\hat{k}_{n, j}^{H}}{\rightarrow} & K\left(A_{n, j}(G / H), n+1\right)
\end{array}
$$

Since $\left(C p_{n, j}\right)^{H}=C\left(p_{n, j}^{H}\right)$ and (by the homotopy excision theorem) $C p_{n, j}^{H}$ is $(n+1)$ connected, we may apply Theorem 3 and find that the $\tilde{k}_{n, j}^{H}$ 's determine a unique $G$-homotopy class from $C p_{n, j}$ to $K\left(A_{n, j}, n+1\right)$. Letting $\tilde{k}_{n, j}$ represent this class, we define

$$
\hat{k}_{n, j}=\tilde{k}_{n, j} \circ i_{n, j} .
$$

Now let $F \hat{k}_{n, j}$ be the homotopy fiber of $\hat{k}_{n, j}$. (See [7] for a proof that this space is $G$-equivalent to a $G$-CW complex.) The composite

$$
X_{n, j+1} \stackrel{p_{n, j}}{\rightarrow} X_{n, j} \stackrel{\hat{k}_{n, j}}{\rightarrow} K\left(A_{n, j}, n+1\right)
$$

is $G$-nullhomotopic since $\hat{k}_{n, j}$ factors through $C p_{n, j}$, so there is a canonical $G$-map $X_{n, j+1} \rightarrow F \hat{k}_{n, j}$ which is a $G$-equivalence (check the fixed point sets) and which makes the diagram

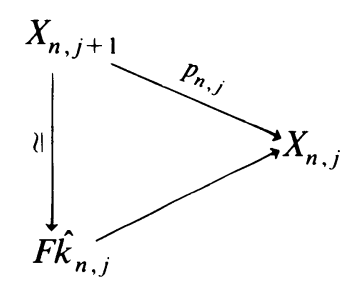

commute.

We now construct a Postnikov tower for $X$ by induction; suppose we have already constructed $G$-spaces $Y_{1,0}=*, Y_{1,1}, \ldots, Y_{n, j}$ and $G$-maps $k_{l, m}: Y_{l, m} \rightarrow K\left(A_{l, m}, l+1\right)$ such that $Y_{l, m+1}$ is the fiber of $k_{l, m}$, and $G$-equivalences $\chi_{l, m}: X_{l, m} \stackrel{\simeq}{\rightarrow} Y_{l, m}$ making the diagram

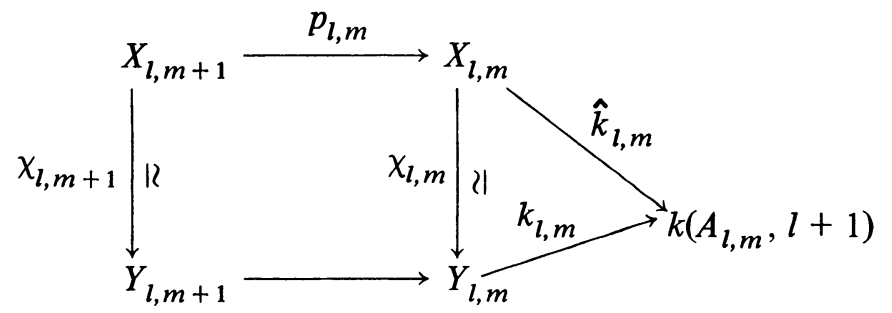


commute. We now construct $k_{n, j}$ and $Y_{n, j+1}$ using the diagram:

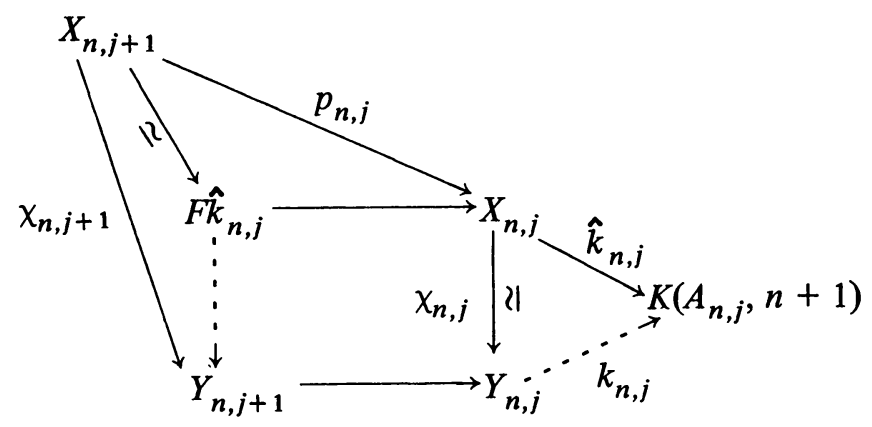

We define $k_{n, j}$ as $\hat{k}_{n, j} \circ \chi_{n, j}^{-1}$ for any homotopy inverse $\chi_{n, j}^{-1}$ of $\chi_{n, j}$. We then define $Y_{n, j+1}$ as the homotopy fiber; the remaining dotted arrow is the induced map on fibers (which can be seen to be a $G$-equivalence by checking the fixed point sets) and $\chi_{n, j+1}$ is then the indicated composite. It is now clear that $X \rightarrow \lim _{\leftarrow} Y_{n, j}$ is a weak $G$-equivalence, as desired.

\section{REFERENCES}

1. G. E. Bredon, Equivariant cohomology theories, Lecture Notes in Math., vol. 34, Springer-Verlag, Berlin and New York, 1967.

2. T. tom Dieck, Transformation groups and representation theory, Lecture Notes in Math., vol. 766, Springer-Verlag, Berlin and New York, 1979.

3. S. Illman, Equivariant singular homology and cohomology, I, Mem. Amer. Math. Soc. No. 156 (1975).

4. J. McClure, Some remarks on Elmendorf's construction (preprint).

5. J. P. May, Classifying spaces and fibrations, Mem. Amer. Math. Soc. No. 155 (1975).

6. G. Triantafillou, Äquivariante rationale homotopietheorie, Bonner Math. Schriften 110, Bonn, 1978.

7. S. Waner, Equivariant homotopy and Milnor's theorem, Trans. Amer. Math. Soc. 258 (1980), 385-405.

DEPARTMENT OF MATHEMATICS, NORTHERn IllinOIS UNIVERSITY, DEKALB, ILLINOIS 60115 